\title{
BLOG@CACM
}

The Communications Web site, http://cacm.acm.org, features more than a dozen bloggers in the BLOG@CACM community. In each issue of Communications, we'll publish selected posts or excerpts.

\section{Inside the Great Wall}

\section{During a trip to China, Jason Hong}

watches for signs of new technologies.

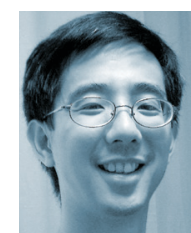

Jason Hong

The Emerging Technology Landscape in China

http://bit.ly/1Rtm2SR

May 25, 2016

I have been in China about two weeks so far, and have been amazed at how much the technology landscape has changed since I last spent time here in 2008. I wanted to share some of these observations because they represent some really unique and compelling uses of technology.

\section{WeChat Mobile Social Networking}

Perhaps the most obvious change is the ubiquity of WeChat, a combination of mobile chat and social networking app. Pretty much every Chinese person I met used WeChat, and I saw people using it in restaurants, on the subway, waiting for the bus, pretty much everywhere. WeChat was developed by powerhouse Tencent, and its features are basically the same as any social networking site: you can add friends, post status updates that friends can see, and send messages to friends. The app feels much cleaner than Facebook's mobile app, though, probably because WeChat started mobile first and as a result had to be very simple.
A cool feature of WeChat is the ability to send virtual red envelopes to others. Real red envelopes are given by close friends and family members on birthdays and Chinese New Year, and contain money. I remember always looking forward to getting these envelopes as a kid. WeChat's virtual envelopes can also be used to transfer money to others, and this feature is already very popular for these special occasions. There is even a Wikipedia page about these virtual red envelopes (http://bit.ly/1r4gwAc) reporting over a billion were sent last year for Chinese New Year.

\section{Mobile Payment Systems}

Another obvious change is the widespread adoption of mobile payment systems. Many people who hosted us for dinner used their smartphones to pay, just by taking a picture of a QR code on the receipt. One person told us how you can use AliPay (from Alibaba, a major e-commerce company), WeChat, or several other apps to buy things from vending machines, again through QR codes. The fact they were compatible was really cool, since I would have expected companies to try to do proprietary systems.

Another person told us how he forgot his wallet at home one day. He almost turned around, but decided to see how well he could do just with his smartphone. It turns out he did perfectly fine, even being able to buy food from street vendors without any problems.

I found the popularity of mobile payment in China amazing, especially since I have yet to directly see anyone in the U.S. use any smartphone mobile payment apps (other than Square, which addresses a different payment space). I have only heard secondhand stories from a few of students at my university about using Venmo or other payment apps.

My best explanation is relative advantage. In the U.S., credit cards are already very popular, and so mobile payment apps only offer a small advantage in this context. In contrast, in China, credit cards were not very common, and so mobile payment systems conveniently filled in the gap, allowing China to leapfrog ahead.

\section{Taxi Hailing Systems}

Didi is a very popular app for hailing taxis. It is only about four years old, and in about half the taxis I was in, the drivers had a holder for their smartphone and were actively using it. One driver even had his app in some kind of active mode, where you could see 
and hear every single nearby request, and it was going off about every $20 \mathrm{sec}$ onds. (I should also note driving safety is a dodgy concept in China. Half the taxis I was in didn't have safety belts, pedestrians walk anytime across the street, and motorcycles often go against traffic.)

Didi used to be two competing taxi hailing companies, but they merged a short while back. I do not know much about Chinese law, but there do not seem to be antitrust laws to prevent large mergers like this. Some people I talked to also said taxi hailing was much better before the merger, since the two companies offered heavy discounts, making it very cheap to get around, but no longer.

Didi is also really interesting when compared with Uber's business model. Unlike Uber, which takes a cut out of the fare, Didi does not seem to; instead, people told me Didi analyzes and sells the data from riders. For example, they can determine origin and destination pairs easily, making it possible to infer demographics and interests of riders. They can then sell that data and even use it for highly targeted advertisements or offers. I was told Didi recently closed a major deal with some banks to do some offers. Apple also announced it will be investing US\$1 billion into Didi, perhaps to help with Apple Pay in China, to improve their maps, and to sell other software and services.

It is also not clear to me how well Uber can do in China. Uber was reported to have lost about $\$ 1$ billion in China last year because of its heavy discounts to win market share. It is not clear to me if Uber China also works with taxis, but Uber's standard model of having everyday people act as drivers might not work as well in China, since the density of taxis in major Chinese cities is already pretty high.

Another interesting story I heard is many of these companies are suffering from fraud. For example, one friend told me how Uber was essentially paying riders to use their service, and how one person had 100 smartphones and colluded with drivers to do fake rides to cash in. In fact, a lot of people commented how all of these discounts were essentially VCs fueling the economy, since a lot of these popular apps were offering deep cuts in prices to gain market share.

Compounding things is the fact that a lot of similar companies exist. Many friends I talked with observed that if you come up with a good startup idea, it does not take very long for copycat companies to appear and offer their VC-funded discounts. One person commented how there used to be a dozen companies that would wash your car overnight (for about $20 \mathrm{RMB}$, or about \$3), but now it has dropped to about two. So competition is very stiff, and it is going to be exciting to see how things play out in the next few years.

\section{Same- or Next-Day Delivery}

If you walk on a major street of any Chinese city, you will likely see a dozen motorized bikes with logos like TMall, JD.com, or even Amazon. Same- or next-day delivery is very common and convenient in China. My wife and I used these services to buy diapers and clothes, and they deliver right to your door. You can also pay on delivery, probably because of the lack of online payment infrastructure, though the mobile payment systems described earlier can also be used.

The density of cities in China is one clear reason these delivery services work. One VC explained another non-obvious reason: in the U.S., retail infrastructure is mostly saturated, meaning e-commerce offers limited relative advantage over what already exists, and often works by cannibalizing brick-and-mortar stores. In contrast, retail infrastructure in China is not as well established, meaning e-commerce has a lot more room for potential growth. These factors, along with relatively low labor and delivery costs, make same- or next-day delivery in China very effective in practice.

\section{Other Odds and Ends}

A few more observations about the tech landscape in China that did not fit in the above categories. One friend told me how the iPhone was sometimes called the "kidney" because one teen literally sold his kidney so he could buy an iPhone; he figured he had two kidneys and really wanted an iPhone. More surprisingly, I was told that for many people, the iPhone costs as much as two months of salary, but I still saw Apple's smartphone almost everywhere.

Chinese people also have a strong affinity for numbers. One interesting quirk is that most telephone numbers in China do not have any dashes or spaces, just a string of 10 or 11 digits. One colleague explained Chinese people have a strong capacity for remembering numbers. However, I can assure readers this is not genetic, given my lack of this facility.

More interestingly, many "holidays" have been created based on special numbers. You may know how Chinese people avoid the number 4 (since it sounds like the word "death" in Chinese) and really like the number 8 (since it sounds like "good fortune"). Riffing on this idea, May 20 (5/20) has been advanced by companies as a new kind of Valentine's Day, since if you say the individual digits it sounds a little bit like "I love you." Yeah, it is sort of a stretch, but WeChat has taken full advantage of this by making it easy to send 520 RMB (about \$75) to one's significant other; there is no option for 5.20 or $52.00 \mathrm{RMB}$. From what I was told, $5 / 20$ was invented just a few years ago, but it has already caught on.

November 11 is another new "holiday," representing single people (11/11, or all 1's). Students I talked to said it was very similar to Black Friday in the U.S., the day of frenzied shopping after Thanksgiving, because what better way is there to celebrate being single than going shopping? Apparently, Alibaba has been breaking sales records every year on this date.

\section{Parting Thoughts}

I hope I have conveyed some of the energy and excitement I have been seeing the past few weeks in China. Even though I am supposed to be on vacation, it is hard to miss all of the innovation and advances everyday people are making use of. I am really looking forward to seeing what the next decade of change will bring.

Jason Hong is an associate professor in the School of Computer Science at Carnegie Mellon University. 